\title{
Development and implementation of a Di-MS based method with full uncertainty estimate to achieve measurement of pharmaceutical residus in natural waters
}

\author{
Sophie Lardy-Fontan ${ }^{1}$, Vincent Brieudes ${ }^{1-2}$, Patrick Candido ${ }^{3}$, Guillaume Couturier ${ }^{3}$, Béatrice Lalere ${ }^{3}$, Hélène Budzinski ${ }^{2}$ \\ Gwenaelle Lavison ${ }^{3}$ \\ ${ }^{1}$ LNE, DMSI, Pôle Chimie Biologie 1 rue Gaston Boissier 75724 PARIS Cedex 15 \\ ${ }^{2}$ Unviversité de Bordeaux, UMR CNRS 5805 EPOC, Allée Geoffroy Saint-Hilaire, 33615 PESSAC CEDEX \\ ${ }^{3}$ Eau de Paris, DRDQE, 33 avenue Jean Jaurès, 94200 Ivry sur Seine
}

\begin{abstract}
The need for effective chemical water monitoring is evident. Indeed the overall management and decision making system driven by the Water Framework Directive (WFD) and other European water policies is strongly dependent on monitoring data. Because the overall decision making process relies, most of the time, on acquired data, it puts considerable pressures on the display of high quality chemical environmental measurements. However, performing fit for purpose measurements implies i) the demonstration of their metrological traceability ii), the evidence of their achievement thanks to accurate and sensitive analytical methods and iii) their statement with a realistic estimate of expanded uncertainty are thoroughly addressed. Concern regarding the presence of potentially active substances in surface waters was brought up by the end of the 90s. Intensive monitoring programs have revealed a chronic state of contamination of water systems by numerous molecules; pharmaceuticals have been highlighted as one of first concern for French aquatic system. This work demonstrates the consequences that (i) the sampling strategy and (ii) methodological and (iii) metrological laboratory choices may have on the measurements' result. It reemphasizes that the main contribution to the uncertainty of measurement is linked to sampling uncertainty
\end{abstract}

\section{Context}

Concerns towards pharmaceuticals in the environment, especially through the water cycle, were brought up in the late 90s [1] and are now laid down in the Article 8c of the WFD (Directive 2013/39/EU). The lingering question of whether the relative low environmental concentration levels of pharmaceuticals would cause adverse effects in humans or wildlife remains unsolved. In France, monitoring programs have clearly emphasised the ubiquity of the contamination by pharmaceuticals from various therapeutic classes: veterinary and human, prescribed and non-prescribed, licit and illicit, and in a wide range of concentrations [2-8].

The need to "display of robust monitoring and methods for a comprehensive assessment of the status of water bodies are essential elements for sound water management" has been reinforced [9]. Accordingly, in addition to accuracy, it implies the display of uncertainty, which is one of the most significant parameters to describe of a measurement result.

Although significant efforts have been made in designing procedures for analytical measurements, very little attention has been paid to the sampling stage. The main difficulties in sampling are to ensure representativity and preserve integrity. Interestingly, many people still consider that the measurement starts when the sample arrives in the laboratory, and undergoes the uncertainty estimation (Figure 1).

To sustain the need of more reliable measurements, this study handled by two laboratories, LNE and Eau de Paris (EdP), focuses on the following points

- Evaluation of the comparability of measurements between laboratories,

- Estimation of overall measurement uncertainty,
- Understanding of sampling uncertainty's main contribution at the station.

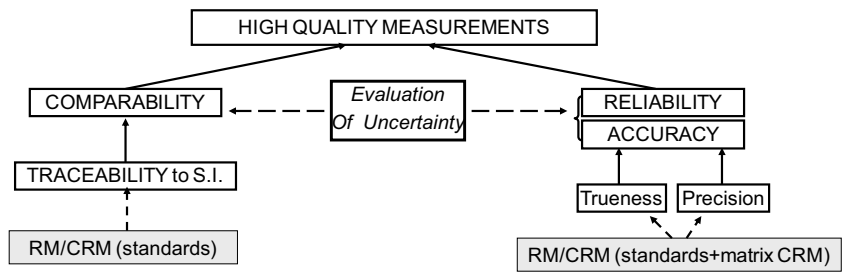

Figure1: Illustration of the components of high quality measurements

\section{Materials and Method}

\subsection{Target analytes}

24 relevant compounds belonging to different chemotherapeutic classes were selected:

- Anticonvulsants and their metabolites: Carbamazepine (CBZ), 10,11-dihydro-10,11-dihydroxy-carbamazepine (DHCBZ), Gabapentine (GPT)

- Non Steroidian Anti Inflammatory Drugs NSAID: Diclofenac (DCF),

- Antidepressants and their metabolites: Citalopram (CTP), Desmethylcitalopram (DCTP), Fluoxetine (FLX), Venlafaxine (VLX), O-desmethylvenlafaxine DVLX),

- Benzodiazepines, "Z-drugs" and their metabolites: Oxazepam (OZP), Prazepam (PZP), Bromazepam (BZP), Zolpidem (ZPD), Zolpidem 4-phenyl-carboxylic acid (AcZ4P), Tetrazepam (TTZP)

- Opioids and their metabolites: Codeine (COD), Norcodeine (NCOD), Tramadol (TMD), EDDP

- Anti-dementia drug: Memantine (MMT)

- Illicit drugs and their metabolites: Cocaine (COC), Benzoylecgonine (BZG), Methadone (METH), MDMA

\footnotetext{
${ }^{\text {a }}$ Corresponding author: author@email.org
} 
Carbamazepine, which is common to both laboratories, is discussed as model.

\subsection{Analysis}

Each laboratory implemented its own analytical methodology: both rely on solid phase extraction (SPE) followed by liquid phase chromatography hyphenated to tandem mass spectrometry [10-11].

Both laboratories characterized the performances of their analytical methods: linearity, recovery, limit of quantification with respects to French national standard NF 90-210 [12]. EdP estimated its measurement uncertainty following NF XP T90-220 (2003) [13]. In turn, LNE estimated its measurement uncertainty according to the GUM approach [14]. Moreover, to ascertain the reliability of interpretation, a strong focus was given to Quality Assurance and Quality Control from the sampling step to the final measurement: implementation of blanks and spiked samples as positive control.

\subsection{Study Design, field operations}

The sampling strategy was designed following the recommendation of the Eurachem Sampling Uncertainty Working Group [15] that define the duplicate method as the simplest and most cost-effective strategy to evaluate uncertainty arising from sampling. The duplicated samples were taken by repeating the same nominal sampling protocol, with permitted variations - bank side, sampling operatives, replication of sampling - that reflect the ambiguity in the sampling protocol and the effect of small-scale heterogeneity of the analytes of interest.

\section{Evaluation of the comparability of measurements between laboratories}

To assess the data comparability, the same samples are regularly analyzed by the two laboratories (EdP and LNE).

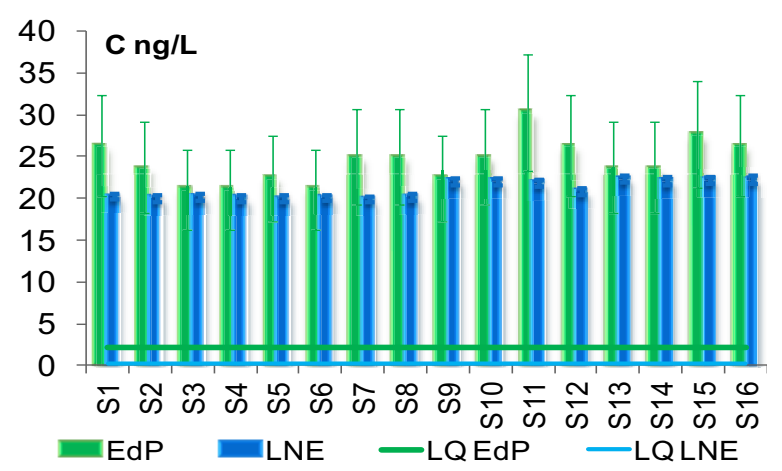

Figure 21 : Histograms of individual measurements of carbamazepine (concentration in ng L-1) by each laboratory. The histograms present the measurement of carbamazepine and its expanded uncertainty $(\mathrm{k}=2)$ in 16 samples by each laboratory.

The concentrations measured by each laboratory do not appear different considering the uncertainties overlap (at
95\% confidence interval) (Figure 2). However, a systematic effect can be observed with [CBZ EdP] > [CBZ LNE]. Each laboratory expresses the concentrations corrected by the absolute recovery whose variation is included in the uncertainty budget. Absolute recovery for carbamazepine is $95 \pm 1 \%$ for $\mathrm{LNE}$ and $76 \pm 8 \%$ for EdP. It is widely recognized that the mastery of recovery is critical to any analytical methodologies. If the correction provides a better approximation of trueness, it can be a significant source of lack of precision. There is currently no regulatory requirement in the environmental field specifying/recommending "best practices". Moreover this information is in many cases not indicated in the protocols or expressed data.

For further reflections, a more precise analysis of the methodological differences between the two analytical methods used has been conducted. LNE implements a method based on isotope dilution/mass spectrometry, the labeled analogues (CBZ D8 for CBZ) are gravimetrically added at the beginning of the sample preparation protocol. EdP implements a pseudo isotope dilution/mass spectrometry, the labeled analogue (CBZ D8 for CBZ) is added volumetrically before instrumental analysis. When implementing ID/MS, better measurement precision can be expected and shall confirm that the use of isotope dilution is a way to better control quantitative analysis in operational monitoring, even if it is more expensive [16].

\section{Estimation of overall measurement uncertainty}

According to the Eurachem strategy, the uncertainties were evaluated and the relative contribution of the sampling and analytical part were calculated.

As highlighted by Figure 3, whatever the parameter, an underestimation of the uncertainty is observed but, the range of this underestimation is broad and depends on the parameter. In fact, the ratio between the measurement uncertainty and analytical uncertainty varies from $1(17 \%$ and $20 \%$ for diazepam respectively) to 13 (4\% and $48 \%$ for benzoylecgonine respectively). In most cases, the contribution of sampling was stronger than the analytical contribution, demonstrating, if necessary, the need of a better definition even a decreasing, if possible.

In the context of monitoring purpose where measurement relies on the succession of sequential steps, measurement should be expressed with an associated expanded uncertainty encompassing the overall procedure, i.e. sampling + analytic.

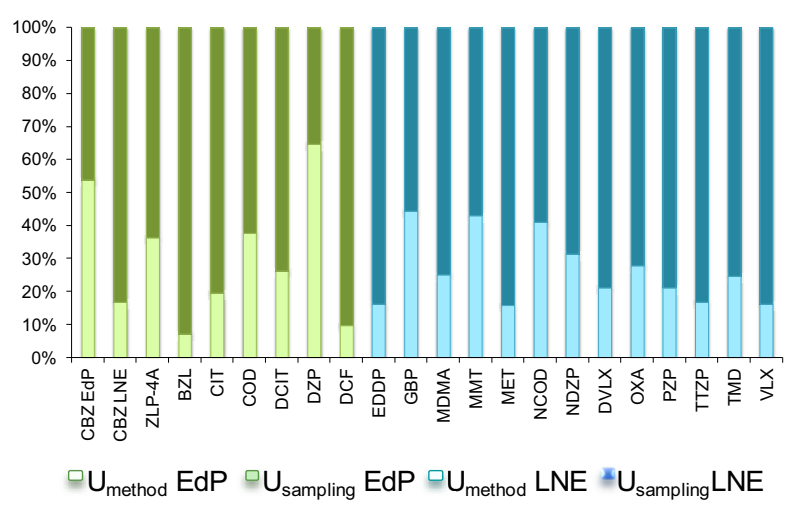


Figure 3: Histograms of the relative contribution of analytic and sampling to the overall measurement uncertainty. Usampling is based on the consideration of the variance of the 16 measurements (whole sets of measurements)

\section{Conclusions}

This work demonstrates the consequences that (i) the sampling strategy and (ii) methodological and (iii) metrological laboratory choices may have on the measurements' result. It reemphasizes that the main contribution to the uncertainty of measurement is linked to sampling uncertainty.

The conclusions of the work re-emphasize the central question of the measurand's definition. It may be different according to the position in the chain of command. When an end-user has to deal with a result expressed in the form ' $\mathrm{x} \pm \mathrm{U}$ ', he will consider that interval as including the range of values attributable to the concentration at the sampling station at a given time. Moreover, he may consider that the uncertainty includes any necessary allowance for heterogeneity of the matrix. On the opposite, the laboratory in charge of the analysis expresses the same concentration result in the form ' $\mathrm{X} \pm \mathrm{U}$ ', it refers to 'the concentration in the laboratory sample analyzed with an interval that includes the ranges of values attributable to the concentration in the provided sample". Obviously, the first includes the effects of sampling, while the second does not. From a metrological point of view, this distinction arises because end-users and laboratories are considering different measurands, respectively 'concentration in the sampling station'/'concentration in the laboratory sample'.

Faced with this ambiguity, a more reasonable question arises: "Are we sure to address the needs of the operational monitoring?"

\section{References}

[1]. Kümmerer, Pharmaceuticals in the Environment, Third ed., Springer, Berlin Heidelberg, (2008).

[2] A. Togola, H. Budzinski, J. Chromatogr A 1177, 150-158 (2008).

[3] E. Vulliet, C. Cren-Olivé, M.F. Grenier-Loustalot, Environ. Chem. Lett. 9, 103-114 (2011).

[4] S. Mompelat, O. Thomas, B. Le Bot, Contamination levels of human pharmaceutical compounds in French surface and drinking water, J. Environ. Monitor. 13, 2929-2939 (2011)

[5] Y. Vystavna, F. Huneau, V. Grynenko, Y. Vergeles, H. Celle-Jeanton, N. Tapie, H. Budzinski, P. Le Coustumer, Water Air Soil Poll. 223, 2111-2124 (2012).

[6]. R. Loos, R.Carvalho, D.C. António, S. Comero, G. Locoro, S. Tavazzi, B. Paracchini, M. Ghiani, T. Lettieri, L. Blaha et al., Water Res. 4, 6475-6487 (2013).

[7] S. Idder, L. Ley, P. Mazellier, H. Budzinski, Quantitative on-line preconcentration-liquid chromatography coupled with tandem mass spectrometry method for the determination of pharmaceutical compounds in water, Anal. Chim. Acta 805 (2013) 107-115.

[8]. L. Vergeynst, A. Haeck, P. De Wispelaere, H.Van Langenhove, K. Demeestere, Chemosphere 119, S2-S8 (2015).
[9] Communication from the commission to the European parliament, the council, the european economic and social committee and the committee of the regions. A Blueprint to Safeguard Europe's Water Resources, COM (2012) 673 final, 24 pages

[10] V. Brieudes, S. Lardy-Fontan, B. Lalere, S. VaslinReimann, H. Budzinski. Accepted Talanta

[11] T. Dinh, G. Lavison, P. Labadie, P. Candido, V. Augustin, F. Alliot, E. Moreau-Guigon, M. Blanchard, M. Chevreuil., Piren Seine

[12] NF T90-210 (2009) Water quality — Protocol for the initial method performance assessment in a laboratory.

[13] NF XP T90-220 (2003) Water quality - Protocol for the estimation of uncertainty associated to an analysis result for physico-chemical analysis method.

[14] GUM - JCGM (2008) Evaluation of measurement data Guide to the expression of uncertainty in measurement.

[15] M.H. Ramsey, S.L.R. Ellison, Eurachem/Eurolab/CITAC/Nordtest/AMC Guide: Measurement uncertainty arising from sampling: a guide to methods and approaches, Eurachem, 2007.

[16] M.J. Capdeville, H. Budzinski,. TrAc Trends Anal. Chem. 30, 586-606 (2011) 